\title{
Análisis de la Sobredemanda del Hospital General de Barrio Obrero. I mplicancias organizacionales y funcionales de una microrred urbana. Asunción, Paraguay 2018-2019
}

\author{
María Stella Cabral de Bejarano ${ }^{1,4}$, Pedro López Puig ${ }^{2}$, Bernardita Villalba ${ }^{3}$, Edgar Giménez \\ Caballero $^{5,6}$, María Clara Bejarano Cabral ${ }^{5}, *$ Liz Rodríguez $^{1}$, * Pablo Retamozo ${ }^{1}$, * Macarena \\ Andino $^{1}, *$ Sandra Alcaraz ${ }^{1},{ }^{*}$ Paola Segovia ${ }^{1}$ \\ ${ }^{1}$ Universidad del Pacifico. Paraguay \\ ${ }^{2}$ Organización Panamericana de la Salud. Paraguay \\ ${ }^{3}$ Instituto de Previsión Social. Paraguay \\ ${ }^{4}$ Centro de Investigación y Proyectos en Sistemas de Salud. CIPSS. Paraguay \\ ${ }^{5}$ Investigación para el Desarrollo ID. Paraguay \\ ${ }^{6}$ Universidad Nacional de Concepción. Paraguay
}

Cómo referenciar este artículo/ How to reference this article:
Cabral-Bejarano MS, López P, Villalba B, Giménez E Bejarano C, Rodríguez L, Retamozo P, Andino M, Alcaraz S, Segovia $\mathbf{P}$. Análisis de la Sobredemanda del Hospital General de Barrio Obrero. Implicancias organizacionales y funcionales de una microrred urbana. Asunción, Paraguay 2018-2019. Mem. Inst. Investig. Cienc. Salud. 2020; 18(2): 12-26

\section{RE S U ME N}

La ampliación del acceso a servicios sanitarios constituye el objetivo intrínseco del Sistema de Salud, cuyos resultados dependen de dinámicas y preferencias de los usuarios y del equilibrio de variables no controlables para alcanzar mejores resultados. Esta investigación evaluativa, cuali-cuantitativa, de corte transversal identifica algunas de estas variables, en un hospital general, cabecera de una micro red de servicios de salud urbana, en Asunción, Paraguay. La unidad de análisis incluyo actores clave ejerciendo roles directivos, gerenciales y asistenciales y usuarios fidelizados a la unidad efectora estudiada. La muestra incluyo 10 directivos, 20 profesionales de salud y 150 usuarios de consultorio externo, urgencias e internados, en tres turnos de atención. Se aplicaron tres cuestionarios conteniendo preguntas semiestructuradas sobre oferta, demanda, organización, supervisión, subsistema de información, comunicación interna, motivos de consulta, tiempos de espera, capacidad instalada, disponibilidad de insumos y medicamentos, motivos de preferencia y algunas variables sociales, epidemiológicas y culturales. Usuarios desconocen la organización de los servicios que integran la micro red urbana, prefieren el hospital general para acceder efectivamente a medios diagnósticos $82 \%$, especialistas, medicamentos y capacidad de respuesta integral $57 \%, 9,9 \%$ posee seguro médico, el $76 \%$ declara empleo formal, $40 \%$ gana más del sueldo mínimo, $95 \%$ de pacientes declaran buena atención en el Hospital General Barrio Obrero, 69 \% está satisfecho. El fortalecimiento de los servicios de primer y segundo nivel puede equilibrar la sobredemanda del hospital cabecera, con implementación de innovaciones organizacionales, incremento de la inversión y adecuados planes de comunicación social.

Palabras clave: Sobredemanda, micro red urbana, hospital general.

Fecha de recepción: Setiembre 2019. Fecha de aceptación: Julio 2020

* Autor correspondiente: María Stella Cabral de Bejarano. Universidad del Pacifico. Centro de Investigación y Proyectos en Sistemas de Salud. Paraguay.

Email: cabralbejarano.mariastella@gmail.com

*Alumnos Investigadores en Formación 


\title{
Analysis of the Excess Demand of the Barrio Obrero General Hospital. Organizational and functional implications of an urban micronetwork. Asunción, Paraguay 2018-2019
}

\begin{abstract}
The expansion of the access to health services is the intrinsic objective of the health system, whose performance depends on user dynamics and preferences and the equilibrium of independent variables to achieve better results. The objective of this study was to evaluate dynamics and factors related to the excess demand in a general hospital, head of an urban micro network in Asunción, Paraguay. This was a qualiquantitative, cross-sectional evaluative study in which the unit of analysis were health actors in directive, managerial, and care providing roles as well as loyal users. The sample included 10 directors, 20 health professionals and 150 users in the areas of consultations, emergencies and hospitalization of three different shifts. Three questionnaires with semi-structured questions on offer, demand, organization, supervision, information subsystem, internal communication, reasons for consultation, waiting times, installed capacity, availability of supplies and medicines, hospital organization, supervision, reasons for preference and some social, epidemiological and cultural variables. The users did not know the organization of the services that were part of the urban micro network. They preferred the general hospital to effectively access to diagnostic means ( $82 \%)$, to specialists, medicines and capacity of integral response $(57 \%), 9.9 \%$ did not have medical insurance, $76 \%$ said they had formal jobs, $40 \%$ earned more that the minimal wage, $95 \%$ said that the Barrio Obrero General Hospital had good attention and $69 \%$ of them were satisfied. The strengthening of first and second level services can balance the excess demand of the main hospital, with the implementation of organizational innovations, increased investment and adequate social communication plans.
\end{abstract}

Keywords: Excess demand, urban micro network, general hospital.

\section{NTRODUCCI ÓN}

La organización de los servicios de salud en redes constituye una estrategia asistencial, sustentada en los principios de equidad y cobertura universal, asumidas como modelo de atención en varios países de América Latina, incluido el Paraguay, mediante la cual se promueve mayor eficiencia en la provisión de servicios, a partir de la ampliación del acceso en el primer nivel de atención y la continuidad e integralidad del cuidado de manera progresiva en los demás niveles, articulando de manera coordinada las unidades efectoras, de diferente complejidad y capacidad resolutiva, en el nivel local, regional y nacional, para lo cual se requiere cualificación y entrenamiento diferenciado del personal y profesionales de salud, desarrollo de lineamientos de políticas de calidad y evaluación continua de procesos y micro procesos orientados a lograr una óptima funcionalidad de las Redes Integradas e Integrales de Servicios de Salud (RIISS), según el nivel valorado ${ }^{(1,2)}$.

Los gobiernos que han elegido las RIISS, han adoptado políticas integrales de Atención Primaria de Salud, y han asumido el compromiso de establecer un modelo de financiamiento, que garantice la disponibilidad presupuestaria, para la adecuación de la capacidad instalada por niveles de atención y complejidad, incluidas las tecnologías apropiadas para cada nivel, la disponibilidad de medicamentos, la dotación de recursos humanos multidisciplinarios para la resolución de problemas de salud prevalentes, con apoyo e integración de recursos comunitarios, del Estado y multisectoriales, para el logro de los objetivos ${ }^{(3)}$.

A nivel global y regional, los cambios demográficos, la ampliación de las metrópolis y concentración del crecimiento urbano, en las últimas tres décadas ha favorecido la migración interna del campo a la ciudad, fenómeno que ha traído consigo el incremento exponencial de la población periurbana, estimada en el 5 al $6 \%$ anual, 
con la consiguiente aparición de problemas sociosanitarios y ambientales, posicionando como prioridad a las políticas de $\operatorname{APS}^{(4,5)}$.

La gobernanza constituye el punto de partida, entre los demás retos multidimensionales necesarios, para transformar los modelos fragmentados y segmentados de los Sistemas de Salud, y avanzar hacia cambios estructurales, posibles solo con generación y asignación de recursos, reconociendo que estos avances son asimétricos en los diferentes países, coexistiendo modelos que funcionan atomizados, en escenarios de alta ineficiencia e inequidad, ejemplificado en colectivos que tienen derechos a más servicios de diferente costo que otros ${ }^{(6)(7)}$.

Las implicancias organizacionales, se interpretan como la manera en que una microrred establece un orden armónico y articulado entre la macro, meso y micro gestión, desarrollando procesos de manera simultánea en todos los niveles del sistema, a través de líneas estratégicas definidas en planes de acción local, adecuadamente coordinados para potenciar la capacidad de respuesta de cada una de las unidades efectoras que la integran, incorporando innovaciones, en un marco de eficiencia técnica y financiera, que garantice la continuidad del cuidado, y un permanentes inputs a la gestión política, económica, gestión clínica, y salud pública ${ }^{(8)}$.

Las implicancias funcionales, se refieren a la adaptación organizacional de acuerdo a las necesidades, estado, nivel de vida y ritmo laboral de las poblaciones, que en el área urbana se caracterizan por la demanda de servicios tangibles, cuantificables y oportunos, que respondan efectivamente a sus expectativas, materializados en servicios ambulatorios, de urgencia, camas de internación, servicios clínicos por especialidades, de odontología, apoyo diagnóstico y medicamentos, variables que organizan las prioridades, redireccionando como componente estratégico las actividades de promoción y prevención en todos los niveles, y con mayor énfasis en el primer nivel de atención ${ }^{(9)}$.

En la última década, variados factores como la gratuidad progresiva de los servicios, el cierre y traslado del Hospital de Clínicas, dependiente de la Facultad de Medicina, genero la ampliación de la demanda hacia el Hospital General de Barrio Obrero de Asunción (HGBO), cabecera de una microrred urbana, que acogió a usuarios procedentes de diferentes barrios de Asunción, ciudades vecinas del área metropolitana, Departamento Central, Chaco Central y otras regiones del país, duplicando la concentración del número de consultas y estableciendo sinergia con unidades efectoras del primer y segundo nivel de atención ${ }^{(10)}$.

Los establecimientos de salud integrantes de la micro red del HGBO, incluyen 21 unidades de salud de la familia, 2 hospitales básicos de mediana complejidad ( $\mathrm{N}^{\circ} 8$ y $\mathrm{N}^{\circ} 11$ ), 1 policlínica de especialidades básicas (Clínica Capellanes del Chaco), todas distribuidas en un radio de 5 kilómetros.

La población asignada al Barrio Obrero de Asunción es de aproximadamente 165000 habitantes, correspondiendo 43607 habitantes (40\%) al HGBO, aunque se han identificado por georeferenciamiento la atención a usuarios que pertenecen a distritos adyacentes (Lambaré, Mariano R. Alonso, Limpio, Fernando de la Mora, Capiatá y Ñemby), barrios de Asunción (Trinidad, Sajonia, Santa Ana, Santa Librada, Bañado Sur, Bañado Tacumbu, San Vicente, San Pablo, San Roque, Dr. Roberto L. Petit, Ricardo Brugada, Republicano, Vista Alegre), compañías rurales del departamento Central, Chaco Central (Presidente Hayes) y otros territorios alejados de la capital.

Evaluaciones cuali-cuantitativas son necesarias para evaluar el contexto de cada territorio, orientado a procesos sistemáticos y continuos de planificación y coordinación para la adecuación de la oferta y capacidad de respuesta a necesidades específicas identificadas localmente.

La conducción de la microrred en estudio responde a dos niveles de gerenciamiento, la Dirección de la XVIII Región Sanitaria y el nivel intermedio subregional u Hospital cabecera, de tercer nivel de complejidad, considerado centro de referencia del territorio o área programática. 
La capacidad instalada del HGBO, entendida como infraestructura, equipamiento y recursos humanos según nivel de complejidad ha venido adecuándose parcialmente, de acuerdo a su nueva categorización, incorporando a los planteles de especialidades básicas (Medicina interna, Pediatría, Ginecobstetricia, Cirugía y Anestesiología), plantel de especialistas en cardiología, traumatología, otorrinolaringología, oftalmología, cirugía plástica, cirugía oncológica, patología cervical, gastroenterología infantil y de adultos, hemodiálisis, dermatología, nefrología infantil y de adultos, urología, psiquiatría, mastología, proctología.

La infraestructura y equipamiento requirió el incremento del número de camas (95), numero de quirófanos, salas de parto, consultorios de especialidades, unidades de cuidados intensivos neonatales, sala de cuidados intermedios y sala de cuidados mínimos, adecuación de salas de internados de adultos y pediátricos, fortalecimiento de servicios de apoyo clínico laboratorial y de imágenes, áreas administrativas (estadística, nutrición, salud pública, y de servicios generales), y mejorar la organización de las prestaciones de salud pública y del sistema de referencia y retorno coordinado con el servicio de emergencia extrahospitalaria (SEME).

\section{Marco conceptual}

El planteamiento teórico de las RIISS, se sustenta en 13 atributos, partiendo de la definición de población a cargo y territorio, amplio conocimiento de necesidades y preferencias que determinan la oferta, extensa red de servicios de salud centrados en la persona, sus familias y comunidades, con componentes de promoción y prevención, gestión clínica y tratamiento, servicios de salud pública, especializados y extrahospitalarios, con enfoque multidisciplinario, existencia de mecanismos de coordinación asistencial entre niveles, que contemple una única gobernanza de toda la red, que promueva la participación social, la cogestión integrada de servicios de apoyo, clínico y logístico, con enfoques de género, interculturalidad y derechos humanos, suficientes, competentes y comprometidos, con un sistema de información integrado, que oriente y sea capaz de discriminar las necesidades de la población, y contribuya a la gestión de incentivos financieros, con énfasis en las comunidades de mayor vulnerabilidad ${ }^{(11)}$.

Las dinámicas de red, aunque no pueden atender todas las necesidades, pueden generar efectos sinérgicos con variedad de sectores y subsectores, a partir de las vivencias y experiencias locales, más allá de los lineamientos teóricos. El trabajo intersectorial contribuye a reconcebir el modelo en zonas urbanas, posicionando la equidad y el derecho a la salud como valor central de la estrategia, subsanando diferencias injustas, garantizando grados de protección financiera y solidaria según las necesidades identificadas.

No existe una formula única, cada país debe labrar su propia ruta, en línea con los Objetivos de Desarrollo Sostenible (ODS), inspiradores de planes de acción local, desde Alma Ata, eliminando progresivamente las barreras ${ }^{(12)}$.

La participación y compromiso de diferentes actores e instituciones locales deben ser permanentemente renovados, asumiendo el liderazgo para analizar, y enfrentar los determinantes sociales de salud específicos del territorio, según sus particulares características, apropiándose de los desafíos administrativos, operativos, logísticos y financieros, integrando la mirada política, económica, social y sanitaria, capaz de potenciar las funciones que son realmente necesarias para resolver los problemas de la población ${ }^{(13)}$.

\section{MATERI AL Y MÉTODOS}

Investigación evaluativa mixta, cuali-cuantitativa de corte transversal, cualitativa preferencial y cuantitativa a pequeña escala.

El componente cuantitativo, fue aplicado a los datos sociodemográficos, y análisis de situación y tendencias mediante porcentajes, integrados de manera coherente con la vertiente cualitativa, en la presentación de los resultados.

En el componente cualitativo, las unidades de análisis constituyeron directivos, gerentes y profesionales de salud, prestando servicios activos al momento del estudio, seleccionados según experiencia y conocimiento del tema, considerando líneas de 
autoridad médica y administrativa, con quienes se coordinó y agendó oportunamente la realización de las entrevistas, respetando el horario laboral.

La muestra incluyo 10 directivos y gerentes y 20 profesionales de salud. Respecto a los usuarios, fueron elegidos aleatoriamente 150 pacientes presentes para consulta en área de consultorio, urgencias y hospitalizaciones en tres turnos de atención (mañana, tarde y noche). Las entrevistas fueron realizadas por miembros del equipo investigador, integrando docentes y alumnos de la materia Administración Hospitalaria del tercer año de la Facultad de Medicina de la Universidad del Pacifico.

Para la recolección de datos se utilizaron tres cuestionarios con preguntas semiestructuradas para los diferentes públicos a entrevistar, construidas a partir de la elaboración de una lista de categorías a priori a explorar.

Los resultados se organizaron, procesaron y analizaron en matrices mediante síntesis de datos de fragmentos discursivos significativos, conceptos, constructos e interpretaciones y explicaciones alternativas, organizados en tablas científicas, generando jerarquías y especificaciones en torno a los aspectos organizacionales y funcionales del HGBO. para demostrar la hipótesis empírica de que la funcionalidad de una microrred urbana requiere el fortalecimiento armónico de sus unidades efectoras, niveles de inversión sostenidos e incrementales, para fortalecer la capacidad instalada, la incorporación de innovaciones organizacionales, la formación de sus recursos humanos, adecuados instrumentos de evaluación y formatos de información, educación y comunicación interna y externa.

Se complementaron los hallazgos con datos procedentes de revisión de la literatura y análisis documental. Se realizó un proceso de metabúsqueda electrónica manual, a partir de descriptores o palabras clave, relacionadas a sobredemanda, organización y funcionalidad de redes de servicios, de bases de datos (Medline, Embase, Lilacs, Cochrane library), se procedió a la catalogación, elaboración de fichas, ordenamiento, análisis crítico, selección de información pertinente y síntesis de hallazgos, relacionada a organización y funcionalidad de redes de servicios, clasificando y seleccionando estudios relevantes de los últimos cinco años.

La generación de salidas correspondiente a la procedencia de cada uno de los usuarios entrevistados se realizó mediante mapeo georreferenciado, utilizando el Software QIS, complementado con análisis parcial de datos en Stata y tablas científicas en Excel.

Se respetaron los aspectos éticos, informando de los objetivos de la investigación, el contenido y tipo de preguntas, el consentimiento individual, y la solicitud de autorización para grabar las entrevistas, asegurando confidencialidad, anonimato, y participación voluntaria.

\section{RESULTADOS}

\section{Distribución georreferenciada de usuarios del HGBO. Encuestados}

Se utilizó el programa QIS, registrando departamento, distrito, barrio y calle exacta del domicilio de pacientes entrevistados, a fin de georreferenciar con exactitud la procedencia, indagando primera consulta, consulta habitual y consulta casual. La concentración observada en las Figuras 1 y $2 a$ y b corresponde a pobladores del Barrio Obrero y barrios circunvecinos de Asunción. Se registraron casos de Villa Hayes y Puerto Casado. 


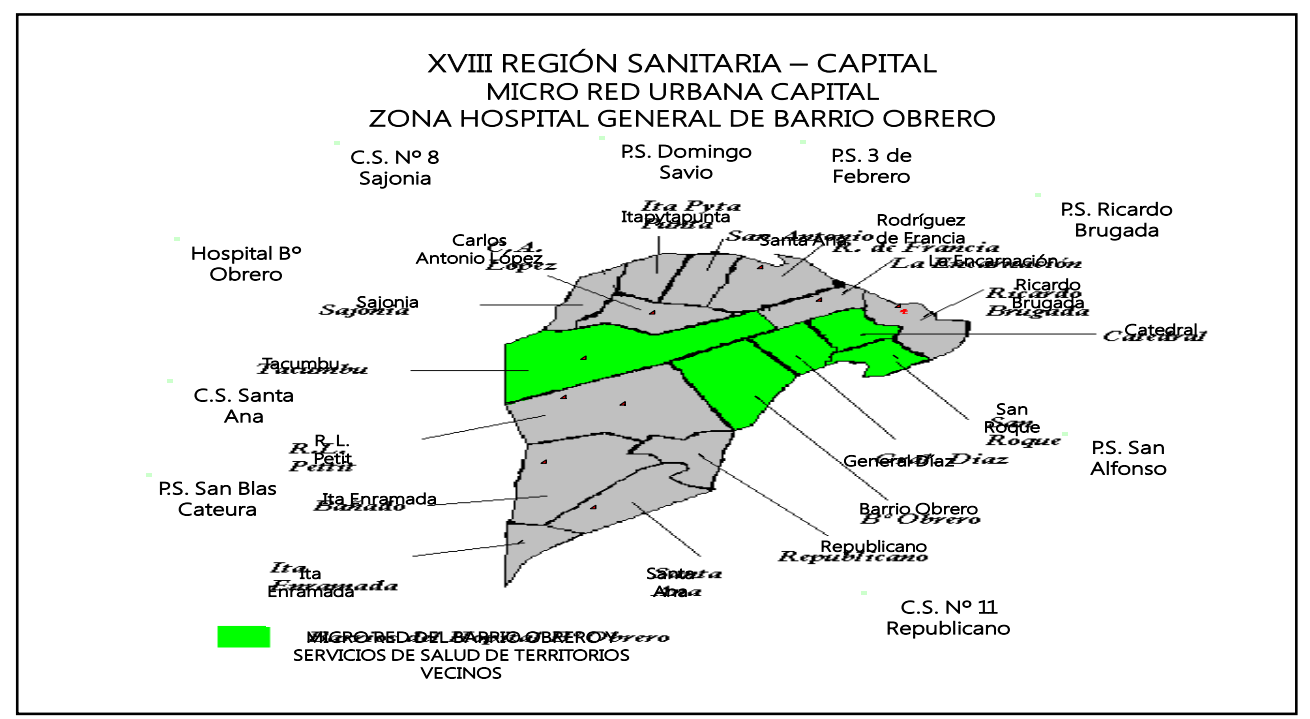

Figura 1: Micro red correspondiente al Hospital General de Barrio Obrero.

Distribución georreferenciada de usuarios del HGBO. según primera consulta, consulta habitual y consulta ocasional. Asunción, agosto de 2018.

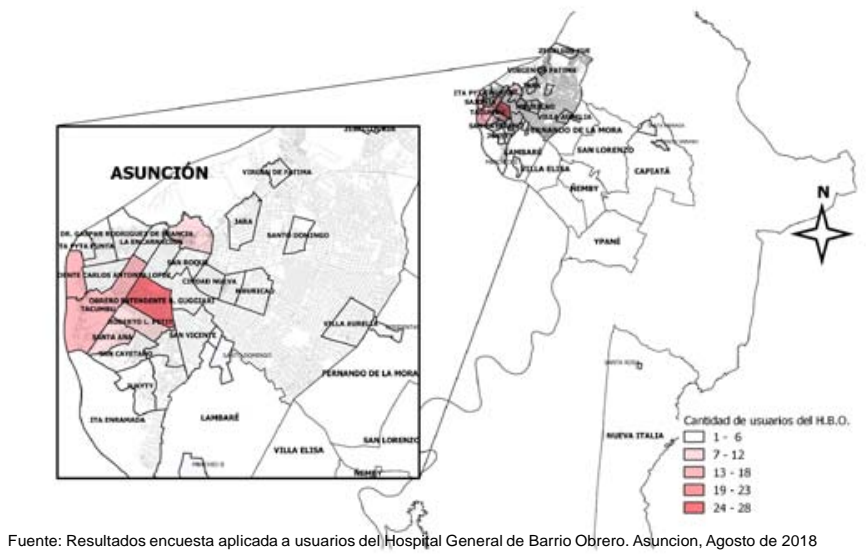

Figura 2a. Caracterización de la Demanda y Rectoría Clínica del HGBO. Georeferenciamiento de usuarios que consultan en el HGBO procedentes del Departamento Central.Asunción-2018

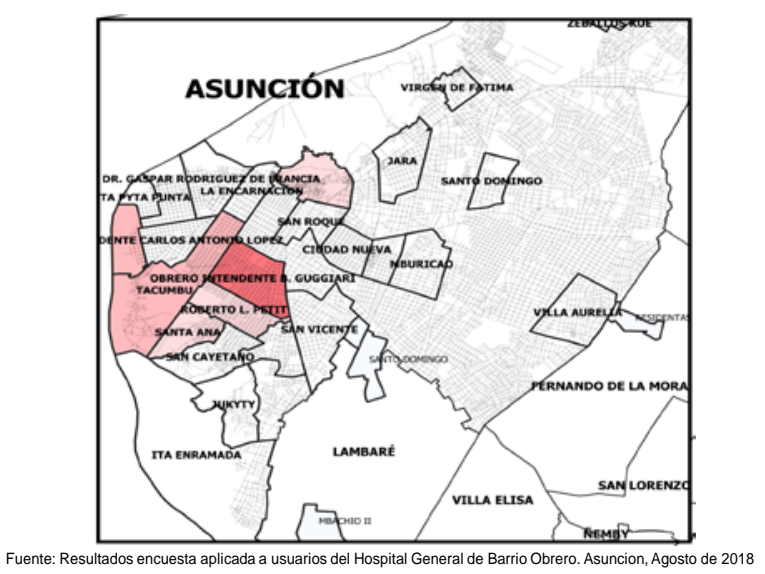

Figura 2 b. Caracterización de la Demanda y Rectoría Clínica del HGBO. Georeferenciamiento de usuarios que consultan en el HGBO procedentes del Departamento Central.Asunción-2018 
Se constató demanda de usuarios de todas las edades, con predominio de $65 \%$ de mujeres jóvenes entre 20 y 39 años, en edad reproductiva, no visibilizándose a la población infantil por aplicarse el cuestionario a usuarios de 15 años y más. Se registró una franja significativa de pacientes con nivel de grado universitario.

El $57 \%$ de los entrevistados declaro preferencia hacia el HGBO, por su capacidad resolutiva a diferencia de otros servicios alternativos de primer y segundo nivel de atención, de la microrred urbana, donde encuentran dificultades para realizar estudios diagnósticos y acceder a medicamentos.

Los pacientes utilizaban 2 a 3 horas para el cumplimiento de los procesos desde que salían de su hogar hasta que accedían efectivamente al servicio solicitado, con excepciones según especialidad.

La mayoría de los usuarios se movilizaban entre las 05.00 y 07.00 a.m. El $3 \%$ de los pacientes tardaba más de una hora en llegar. Se observó una curva de incremento de demanda a partir de las 11.00 a.m. que correspondía a los usuarios que consultaban por la tarde.

El $35 \%$ de los pacientes tardaba 30 minutos a una hora en llegar, procedentes de barrios de la capital y distritos vecinos del departamento central.

El $82 \%$ de los encuestados manifestó su preferencia por las facilidades de accesibilidad. El 27,33\% de usuarios llegaba al servicio caminando y $56 \%$ en transporte colectivo.

La demanda demostró incrementos en los tres turnos, de 08.00 a 10.00 a.m., una meseta o segundo incremento a las 11.00 a.m. En relación con el tiempo que tardaba el paciente en ser atendido, el $72 \%$ de usuarios coincidió que el tiempo de espera promedio era de 1 y 2 horas.

Acerca de las condiciones económicas de los usuarios, el $61 \%$ de la población entrevistada trabajaba regularmente, de este grupo el $76 \%$ refirió empleo formal, 33 $\%$ son jornaleros. El 40 \% ganaba más del salario mínimo y entre los que ganaban salario mínimo y más del salario mínimo se constató un $75 \%$ de usuarios que deberían acceder al seguro social, aunque solo el 9,9\% de los encuestados poseía algún tipo de seguro médico.

El 34,67 \% de los entrevistados declaró gastos de traslado al hospital de alrededor de 10000 guaraníes ( 2 U\$), un 3\% declaró cifras superiores mientras que el $36 \%$ no contestó la pregunta. Los gastos de hospitalización (internación personal o de familiares) fueron estimados entre 25000 guaraníes (5 U\$) hasta un millón de guaraníes (200 U\$), dependiendo del tipo de enfermedad o procedimiento realizado.

Se utilizó la mediana para medir gastos de transporte, de 20000 guaraníes (4 U\$), hospedaje 100000 guaraníes ( $20 \mathrm{U} \$$ ) y gastos varios alrededor de 50000 guaraníes (8 U\$). Solo en un caso, una paciente declaró gasto de transporte de 160000 guaraníes ( 26 U\$) y de hospedaje de 280000 guaraníes (30 U\$).

El $82,67 \%$ de los pacientes encuestados podían costear estudios diagnósticos solicitados por los profesionales del servicio, aquellos no incluidos en los servicios de apoyo diagnóstico declarados gratuitos. El $38 \%$ de los pacientes manifestó respaldar gastos adicionales complementarios con sus propios recursos al momento de la encuesta. El $100 \%$ de los encuestados refirió carecer de un seguro médico.

El $82,67 \%$ de usuarios, coinciden con directivos y gerentes sobre la necesidad inaplazable de ampliar infraestructura, tecnología y numero de recursos humanos, para reducir las listas de espera en contraste con la manifestación del 95,43\% de usuarios que manifestaron satisfacción y percepción de calidad en la atención, facilitada por personal y profesionales de salud.

El 95,33 \% resalto la organización del servicio, el $86 \%$ lo recomendaría a otros pacientes y el $69 \%$ se mostró satisfecho respecto a las orientaciones recibidas por el personal administrativo ante consultas sobre procedimientos a seguir dentro de la institución.

El $6,4 \%$ de usuarios se orientó por carteles indicadores. Se registró $21.4 \%$ de no respuesta. Sobre los servicios de preconsulta, el $40 \%$ respondió que no se les pesó, no se les tomó la temperatura y no se les brindó charlas educativas. 
Las Tablas 1, 2 y 3 contienen fragmentos discursivos y algunas síntesis reflexivas, procedentes de las entrevistas a los actores clave de nivel directivo, profesionales y usuarios.

Tabla 1: Observaciones de directivos, gerentes, profesionales y personal de salud sobre rectoría clínica y sobredemanda de atención del HGBO, 2018

\begin{tabular}{ll}
\hline Dimensión & Subdimensiones \\
& \\
\hline CLINICA DEL & Factores relacionados \\
HOSPITAL & a la sobredemanda \\
GENERAL DE & \\
BARRIO & \\
OBRERO, &
\end{tabular}

Infraestructura y equipamiento

Dotación de RRHH (Especialistas vs generalistas). Análisis de dotación y carga horaria

Medicamentos e Insumos

Organización

\section{Percepciones de Directivos, gerentes, profesionales y personal de salud sobre Rectoría Clínica, y factores vinculados a oferta sobredemanda del HGBO.}

Elevada población asignada, dotación de plantel de
profesionales médicos de alta competencia. Organización adecuada. Disponibilidad de horarios escalonados.

Preferencia espontanea de la población que acude al HGBO por la capacidad de respuesta a las necesidades de salud de la población asignada y fuera de zona.

Innovación de tecnologías insuficiente. Limitaciones de oferta de medios diagnósticos. Productividad puede ser incrementada con mayor inversión y dotación de equipos

Mecanismos regulatorios, fiscalización, supervisión, deben incrementarse, ajustando cronogramas de atención para 24 horas.

Equipos especiales como gasómetro, auto analizadores químicos, máquinas para dosaje de electrolitos requieren mantenimiento y renovación.

Predominio de atención de médicos familiares, médicos generales, clínica médica, gineco-obstetras y pediatras.

Necesidad de redistribución e incremento de horarios de atención de especialistas: traumatólogos, cardiólogos, otorrinolaringólogos y cirujanos.

Equilibrar inequidad de volumen de trabajo y productividad entre especialidades. Ejemplo: Psiquiatría, Cirugía Plástica

Especialistas contratados y permanentes deben conocer las políticas, metas, objetivos, programas y proyectos del Ministerio de Salud Pública y Bienestar Social.

Se debe incorporar programa de desarrollo de recursos humanos, incluyendo formación en aspectos de bioética, valores, relaciones humanas e interculturalidad.

A pesar de limitación de recursos, usuarios afirman que profesionales están comprometidos y demuestran alto sentido de solidaridad para resolver problemas.

Más del $70 \%$ de encuestados denuncio problemas de abastecimiento de suministros básicos y desempeño sin materiales, insumos e instrumentos adecuados.

Atención está garantizada en horarios escalonados de 07.00 a 19.00 horas. Existen refuerzos en tiempos de contingencia (inundaciones y epidemias, vacaciones).

El $100 \%$ de encuestados manifiesta buena la organización del HGBO., aunque el espacio físico disponible es restringido para ampliar innovaciones.

Turnos especiales (turno noche) deben ser fortalecidos.

Demandan fiscalización de cumplimiento de carga horaria de especialistas, redistribución de funciones según carga horaria. Áreas específicas deben ser intervenidas y asignadas según idoneidad, como el departamento de estadísticas.

Las unidades efectoras de la microrred deben ser reguladas y fiscalizar su funcionamiento para reducir la sobredemanda del nivel especializado. 


Servicios de Salud
Publica en la
microrred

Pluriempleo

Preferencia de turnos de atención de usuarios

Supervisión

Subsistema de información Insuficiente implementación de programas de promoción y
prevención en el primer y segundo nivel de atención.

Potencialidad para establecer sinergias con entidades cooperantes, para construir gobernanza local para el desarrollo de actividades de apoyo.

Se ejerce contraloría social, que contribuye a ajustar la oferta de servicios y sostenibilidad de programas.

Baja coordinación de programas de salud pública con líderes de la comunidad, y referentes del municipio.

El $80 \%$ de los encuestados trabaja en otras instituciones del sector privado y seguridad social.

Mayor demanda en el turno mañana y tarde. Horarios con mayor número de profesionales. Presencia de jefes de sala y de departamento., entre ellos

Las amas de casa se movilizan con mayor facilidad en horas de la mañana. El turno tarde es preferido trabajadores formales e informales.

Elevado número de pacientes con trabajo formal, que no reciben los beneficios del seguro social.

Más del $60 \%$ de encuestados, afirma que las supervisiones son esporádicas.

Un funcionario menciono la realización de evaluaciones cada 3 meses, se realizan evaluaciones conjuntas para la revisión de cumplimiento del POA anual.

Una persona afirmo que el área de enfermería tiene adecuada supervisión

Un gerente de área afirma que el acceso a la información es limitada, está fragmentada y dispersa. No se realizan controles de gestión conjunta.

La independencia de cada departamento incide en la disponibilidad de información para evaluar procesos y realizar controles de gestión específicos.

Fuente: Elaboración propia, equipo investigador. Análisis de la Sobredemanda del Hospital General de Barrio Obrero.
Implicancias organizacionales y funcionales de una microrred urbana. Asunción, Paraguay 2018-2019

Tabla 2: Dimensiones y subdimensiones de Rectoría Clínica, HGBO 2018 - 2019

Dimensión Subdimensiones Respuestas sobre calidad. HGBO.

Disponibilidad de Desconocimiento de normas de referencia y contrarreferencia entre niveles

RECTORIA normas, guías y de atención.

CLINICA protocolos Asimétrico desempeño en la aplicación de normas, guías y protocolos por

DEL HGBO. directivos y profesionales.

Demanda de difusión y capacitación del nuevo Manual de Organización de los Servicios en el marco de las RIISS.

Deficiencias de comunicación interna, entre servicios y entre los centros de regulación, que incide en la efectividad y oportunidad para agilizar Dificultades de derivación de pacientes críticos a hospitales especializados, por déficit de camas de terapia intensiva.

Definición de Los tiempos de espera en atención ambulatoria están sujetos a la tiempos de especialidad y flujo de pacientes y no están regulados. Pacientes acceden a espera consulta por orden de llegada.

Las consultas se agendan a las 06.00 para turnos mañana y tarde y a partir de las 11.30 horas para turno tarde y noche.

Los profesionales llegan 2 horas después de los agendamientos. Existe demanda de incorporación de turno siesta. La ampliación depende de la La demanda de determinadas especialidades como Pediatría y Clínica Médica condiciona razonables tiempos de espera. A diferencia de otras Se estima que los tiempos de espera en urgencias, clasificados mediante el R.A.C. (Recepción, Acogida y Clasificación), son similares a cualquier 


\begin{tabular}{|c|c|}
\hline & $\begin{array}{l}\text { En especialidades con pocos profesionales y alta demanda (neonatología, } \\
\text { neurología infantil, psiquiatría, cirugías programadas otros) los tiempos de } \\
\text { espera aumentan. }\end{array}$ \\
\hline \multirow[t]{3}{*}{$\begin{array}{l}\text { Dotación de } \\
\text { RRHH }\end{array}$} & $\begin{array}{l}\text { La dotación de RRHH es adecuada, pero la productividad desigual. } \\
\text { Asimetría de productividad entre generalistas y especialistas. }\end{array}$ \\
\hline & $\begin{array}{l}\text { Existe sobrecarga en clínica médica, medicina familiar, pediatría, gineco- } \\
\text { obstetricia. } \\
\text { Existen profesionales de determinadas especialidades que no cumplen } \\
\text { adecuadamente la carga horaria asignada. }\end{array}$ \\
\hline & $\begin{array}{l}\text { Especialidades como cirugía plástica, requieren cirugías programadas } \\
\text { discontinuas. }\end{array}$ \\
\hline \multirow[t]{3}{*}{$\begin{array}{l}\text { Comunicación } \\
\text { interna }\end{array}$} & $\begin{array}{l}\text { Se percibe insuficiente e inadecuada, y esta brecha contribuye a la } \\
\text { fragmentación de la atención. }\end{array}$ \\
\hline & $\begin{array}{l}\text { La dotación de insumos informáticos, equipos y nuevas tecnologías deben } \\
\text { ser incrementados en todas las unidades efectoras de las redes sanitarias }\end{array}$ \\
\hline & $\begin{array}{l}\text { Contratación de expertos para mantenimiento de equipos y tecnología son } \\
\text { necesarios en la programación y objetos de gasto a considerar. }\end{array}$ \\
\hline \multirow{4}{*}{$\begin{array}{l}\text { Propuestas de } \\
\text { mejora } \\
\text { identificadas por } \\
\text { directivos, } \\
\text { gerentes y } \\
\text { personal de salud }\end{array}$} & $\begin{array}{l}\text { Prioridades identificadas en la reorganización de las áreas de admisión: } \\
\text { agilizar las dinámicas de acceso a la consulta. }\end{array}$ \\
\hline & Modernizar el área de estadística y archivo. \\
\hline & $\begin{array}{l}\text { Ampliar disponibilidad de consultorios para la atención ambulatoria, } \\
\text { soportadas por profesionales de las especialidades básicas más } \\
\text { demandadas y médicos familiares. }\end{array}$ \\
\hline & $\begin{array}{l}\text { Mayor comodidad y confort las salas de espera (sillas, bancos, bebederos, } \\
\text { elementos de aseo, jabón, papel higiénico, toallas de papel para secado de } \\
\text { manos). }\end{array}$ \\
\hline
\end{tabular}

Fuente: Elaboración propia, equipo investigador. Análisis de la Sobredemanda del Hospital General de Barrio Obrero. Implicancias organizacionales y funcionales de una microrred urbana. Asunción, Paraguay 2018-2019 
Tabla 3: Oportunidades de mejora, Hospital General de Barrio Obrero. Asunción, 2018 - 2019

\section{Dimensión Subdimensiones Respuestas sobre oportunidades de mejora}

Oportunidades Fortalecimiento de la La asignación y ampliación de espacios para el desenvolvimiento de programas prioritarios de salud pública De Mejora capacidad instalada requieren ampliación de infraestructura.

El HGBO. ya no dispone de espacio físico para ampliar sus instalaciones, lo que plantea la construcción de una nueva sede.

Incrementar la dotación de Recursos Humanos en todas las unidades efectoras de la microrred urbana de Barrio Obrero. con énfasis en la Atención Primaria

Incrementar el número de consultorios para hacer frente a la demanda

Incrementar la dotación de insumos y equipos informáticos y los medios para hacer más efectiva la comunicación

Reorganización de subsistemas de apoyo
Reorganización de áreas de admisión (acceso a la consulta), respondiendo a prioridades identificadas (agilizar las dinámicas de acceso a la consulta).

Ampliar y modernizar el área de estadística y archivo, considerada estratégica para el análisis de gestión y cumplimiento de metas

Ampliar disponibilidad de consultorios para la atención ambulatoria,

Incrementar el número de profesionales, de las especialidades más demandadas (médicos familiares, clínicos pediatras y gineco-obstetras)

Mayor comodidad y confort las salas de espera (sillas, bancos, bebederos, elementos de aseo, jabón, papel higiénico, toallas de papel para secado de manos)

Supervisión Monitoreo y

Evaluación
Actividades de supervisión esporádicas, a cargo de personal que cumple paralelamente otras funciones. Se necesita personal idóneo para esta función

Ausencia de sistematización de resultados de monitoreos rápidos, con la consecuente ausencia de planes de mejora

El nuevo Manual de Organización de los Servicios en el marco de las RIISS, propone nuevos instrumentos de monitoreo rápido y supervisión de servicios.

Se precisan herramientas alternativas para identificar eslabones de mejora por servicios y departamentos

No se dispone de personal competente para supervisión, monitoreo y evaluación de procesos

Asignar roles de supervisión a equipos de supervisión, multidisciplinarios entrenados, tratándose de una actividad de alta competencia 


\section{DISCUSIÓN}

Se consideraron dos vertientes de análisis, por un lado, las observaciones emergentes en torno a la funcionalidad de la microrred urbana, y por otro el análisis de los factores que generan la sobredemanda en el hospital general cabecera.

Los hallazgos reflejan la vivencia y comportamiento de una población, caracterizada por una intensa vida laboral, integrada por familias de bajos y medianos ingresos, trabajadores formales e informales. En ese contexto, desde hace más de una década, se inició la readaptación y planificación de unidades efectoras de primer y segundo nivel de atención, integrando una microrred, orientada a brindar cobertura progresiva a una población urbana con una de las tasas de crecimiento más elevadas del área metropolitana, aunque la evolución de implementación progresiva de los diferentes atributos de las RIISS promovidos al principio para articular funcionalmente los servicios de salud del territorio de acuerdo a un modelo coherente y con visión estratégica orientada a disminuir la fragmentación del sistema, fue interrumpida en el transcurso de cambios de gobierno y de procedimientos administrativos, debilitándose el objetivo primordial de resolver en el primer y segundo nivel de atención el $80 \%$ de los problemas e incrementar el número de personas sanas.

La redefinición de conceptos dirigidos a superar el modelo biomédico y hospitalocentrico, ha logrado en la última década avances hacia un sistema más integral e integrado, reconociendo inadecuaciones y asimetrías que persisten relacionadas a los niveles de inversión, financiamiento y compromiso de las comunidades, los actores políticos y sociales y los profesionales de salud.

Existen claras diferencias de funcionalidad en las microrredes del área urbana y rural, relacionadas a variables administrativas por un lado (barreras financieras, capacidad instalada) y variables no administrativas (contextos socioeconómicos y culturales específicos, dificultades de información y comunicación entre usuarios y equipos sanitarios, desconocimiento sobre las prestaciones y cartera de servicios disponible según niveles de atención, además de escalas y tamaños de las poblaciones), como lo refieren Hirmas y Aday, $2013^{(14)}$.

Algunos hallazgos muestran que las evaluaciones se basan en resultados estadísticos, análisis epidemiológicos e indicadores de productividad, no constatándose resultados de evaluaciones cualitativas relacionadas a la dimensión social y sanitaria con enfoque de red. Falta debate y análisis conducentes a la implementación de cambios organizacionales y funcionales que garanticen la continuidad del cuidado.

El nuevo Manual de Organización de los Servicios en el marco de las RIISS, propone instrumentos de evaluación, monitoreo rápido de los servicios y controles de gestión periódicos con participación de los actores que integran los cuerpos de gobernanza local, con periodicidad normada a fin de dar el seguimiento necesario a la evaluación territorial en su conjunto ${ }^{(15)}$.

La conducción territorial, ha tenido como fortaleza la disponibilidad de normas operacionales, aunque se ha constatado debilidades en el manejo del sistema de información y comunicación y de otros subsistemas compartidos relacionados a la gestión clínica, el desarrollo de los recursos humanos, y la coordinación para ampliar redes de diagnóstico, transporte e insumos y medicamentos.

Ningún estudio evaluativo consultado, o experiencias compartidas demuestran que algún país ha logrado instalar todos los atributos para la implementación de las RIISS, reconociendo que el desarrollo de los diferentes componentes, son asimétricos debido a restricciones relacionadas con realidades y dificultades específicas.

Vargas, I et al, 2010 enfatizan la importancia de ubicar donde y en qué momento se presentan las mayores barreras no administrativas dentro de los establecimientos de salud, para identificar con mayor claridad las alternativas de solución. En este estudio se ha identificado que las competencias y el entrenamiento técnico constituyen una de las dimensiones de mayor importancia, junto al análisis continuo de los datos estadísticos de gestión, monitoreo, vigilancia e implicancias organizacionales ${ }^{(16)}$.

Las microrredes urbanas requieren subsidios especiales para la implementación de iniciativas y estrategias diferenciadas de fortalecimiento, orientadas a incrementar la funcionalidad, incorporando innovaciones organizacionales, como elaboración de 
escalas de trabajo, implementación de espacios formales de intercambio de información entre unidades efectoras, reorganización de consultorios de especialidades, y refuerzos en la dotación de recursos humanos que den soporte a la atención ambulatoria e internación de pacientes.

La coordinación asistencial no ha sido congruente, con las necesidades de los usuarios, que buscan diferentes especialistas de manera fragmentada, para atender problemas aislados, no lográndose la continuidad del cuidado, debido a que los equipos de salud no interactúan en los procesos. Para lograr la interacción entre niveles, deben concretarse prácticas periódicas de entrenamiento conjunto de médicos de familia, generalistas y especialistas, que contribuyan a mejorar el desempeño integrado de los equipos sanitarios entre niveles de atención, estableciendo condiciones para la innovación, a través de iniciativas evaluables y reproducibles, mediante experiencias exitosas o de benchmarking, considerando la toma de decisiones informadas en evidencia, alimentadas por el análisis de estudios de caso, análisis de redes locales e investigaciones operativas ${ }^{(17,18)}$.

La segunda vertiente de análisis se ha enfocado en la identificación de factores que generan la sobre demanda del hospital cabecera de red.

El modelo de conducción no ha logrado constituir un cuerpo de gobernanza, integrado por actores del nivel nacional, regional y local, para implementar satisfactoriamente los cambios y transformaciones, debido a recambios e interrupciones en los últimos tres periodos de gobierno, predominando el modelo biomédico y hospitalocéntrico, en torno al Hospital General de Barrio Obrero.

Las limitaciones de inversión han retrasado la ampliación de la capacidad instalada, de acuerdo con la cartera de servicios definida por niveles, repercutiendo directamente en el incremento del gasto de bolsillo de los usuarios, en objetos de gasto como transportes, traslados, adquisición de medicamentos especiales, estudios diagnósticos complementarios y días adicionales de hospitalización, que refuerzan resultados de investigaciones previas que afirman que el financiamiento del Sistema de Salud del Paraguay es predominantemente privado, y la protección financiera es inequitativa y débil, habiendo variado de 53,0\% del gasto total en salud en el 2000 a tan solo $49,3 \%$ en el 2014 según Giménez Caballero y colaboradores, $2017^{(19)}$.

De todas formas, la fidelización de los usuarios de todas las edades, al HGBO, se atribuye a la atención optima e integral, buen trato, calidez, humanismo en la atención y disponibilidad de servicios diagnósticos y terapéuticos acordes a los estándares previstos, coincidiendo con Del Carmen Sara y Soto y colaboradores, $2019^{(20)}$, quienes se refieren a la adhesión que genera la percepción de calidad y excelencia profesional, y la capacidad real para resolver problemas de mayor complejidad, como lo asevera Ugarte-Ubilluz, 2019 ${ }^{(21)}$.

Un solo indicador, relacionado al cumplimiento de la carga horaria asignada a los profesionales, identifica desempeño y remuneración desigual, al mismo tiempo que revela la debilidad de procesos regulatorios y organizacionales, traducida en productividad inequitativa e ineficiente, no atribuido a la cualificación individual.

La disponibilidad de normas, protocolos y guías se consideran una fortaleza de la gestión, pero son insuficientes los procesos formativos y entrenamientos de los equipos sanitarios.

Los medios de comunicación interna y externa tienen gran potencialidad para incorporar cambios orientados a dar respuesta a prioridades como las dinámicas de acceso y el desarrollo de programas preventivos prioritarios de salud pública. Fernández Lorenzo, 2017(22), maximiza la importancia de estrategias persuasivas, detallistas y efectivas del marketing social en salud, orientadas en el caso del HGBO a incrementar el conocimiento de las dinámicas organizacionales y el conocimiento de la cartera de servicios que prestan las unidades efectoras de primer y segundo nivel de atención a partir de diagnósticos locales participativos, y con apoyo de organizaciones públicas, sociales, empresariales, organismos internacionales y recursos de gobiernos descentralizados que contribuyan a las actividades y programas de promoción y prevención, reconociendo la efectividad de actividades sociales y recreativas, 
campañas sociales explicativas, ruedas de prensa, vallas publicitarias, afiches, construcción de redes comunitarias de apoyo social.

La preferencia por el acceso a las consultas de urgencia en detrimento de las consultas programadas en el hospital especializado, traduce la elección de vías rápidas, para resolver necesidades inmediatas, situaciones que refuerzan la necesidad de incrementar la comunicación, como potencial contribuyente para lograr eficiencia con especialistas, orientan sobre ajustes relacionados a la eficiencia, como lo manifiestan Restrepo y colaboradores, 2014, que promueven el manejo de indicadores de accesibilidad física, distancias, tiempo invertido, costos de transporte, capacidad individual para solventar la atención y satisfacción con la respuesta obtenida, para planificar la oferta y establecer nuevas relaciones funcionales y preferencias hacia servicios de primer y segundo nivel de atención ${ }^{(23) .}$

Hirmas Aday, 2013 menciona entre los facilitadores, los horarios extendidos, diurnos, vespertinos y nocturnos, y la disposición y respuesta a necesidades diferenciadas de trabajadores según la distribución de sus horarios laborales.

Para lograr la satisfacción tanto de usuarios como de prestadores, se requiere que todos los recursos actúen en conjunto para generar resultados de salud, medibles y observables, aunque el rol del médico es considerado como el principal en los equipos sanitarios, quien se ve interpelado a ampliar y reorientar la atención clínica en un entorno de patologías sociales y ambientales, alcoholismo, toxicomanía, prostitución, delincuencia, violencia, abandono de madres y niños, enfermedades mentales y crónico-degenerativas características de las grandes ciudades ${ }^{(24,25)}$.

La sobredemanda del H.G.B.O. constituye la consecuencia de una red limitada en su funcionamiento, y el reflejo de necesidades diferenciadas de un colectivo urbano metropolitano.

La RIISS urbana, requiere continuos ajustes para la implementación efectiva de una atención integrada, en contextos locales específicos, con adaptación cultural y entrenamiento permanente de los equipos sanitarios.

La segmentación es un defecto del sistema y la fragmentación un problema de organización gestión y financiamiento, sobre el cual se puede incidir.

El predominio del modelo biomédico debe ser progresivamente reemplazado por modelos organizacionales de redes integradas de servicios adaptadas con mayor precisión en zonas urbanas para la atención de particularidades y especificidades.

La reducción de la fragmentación y el progreso hacia la integración y articulación de servicios requieren fortalecimiento de las dimensiones de calidad, eficiencia, eficacia y efectividad, con visión sociohistórica, actualización de marcos legales, normativos y organizacionales y ajustes continuos de planificación e inversiones.

Las oportunidades de mejora de la calidad de atención como derecho para todos los ciudadanos dependen del incremento del financiamiento y la superación de la burocracia administrativa que restringe la inversión en sus diferentes dimensiones.

Los avances están condicionados por la adecuación del modelo organizacional local, con participación de la comunidad, incorporando líneas claras de política, adecuadas en su marco legal, fortalecer el financiamiento, para corregir las ineficiencias internas en el uso de los recursos, apoyar liderazgos de nivel nacional, regional y local para convocar y generar cambios y transformaciones, apoyadas en instrumentos de evaluación innovadores, maximizar las estrategias de información, educación y comunicación, como aliados para transformar, educar, organizar y regular la funcionalidad de la microrred de Barrio Obrero y su hospital cabecera, promover la participación inclusiva de la comunidad, y asegurar la periodicidad de entrenamientos y actividades formativas, dirigidas a profesionales y personal de salud, además de la realización de investigaciones operativas, que contribuyan a identificar con claridad, las brechas organizacionales y funcionales, para redireccionar objetivos y orientar transformaciones substanciales, conducentes a evitar la fragmentación de unidades efectoras que operan de manera independiente sin integración. 


\section{AGRADECI MI ENTOS}

Los autores agradecen a las personas e instituciones que han colaborado brindando información y aportando sus opiniones para este estudio. El trabajo contó con financiación de la Universidad del Pacifico Privada, la colaboración del Hospital General de Barrio Obrero, y profesionales multidisciplinarios ejerciendo roles directivos, gerenciales y de atención directa en el HGBO. Asimismo, se agradece al Dr. Pedro López Puig, Consultor de OPS/OMS, Consultor Senior de Servicios de Salud en Paraguay, por su participación en el análisis y discusión de los resultados.

\section{REFERENCI AS BI BLI OGRÁFI CAS}

1. Alum N, Cabral-Bejarano, M.S. Sistema de salud de Paraguay. Rev Salud Pub Parag 2011; Vol. 1 (1): 13-25

2. Organización Panamericana de la Salud. Redes integradas de servicios de salud: herramienta de valoración del proceso de integración de redes de servicios. Washington, DC: OPS; 2016.

3. Dullak Roberto, Rodriguez-Riveros María Isabel, Bursztyn Ivani, CabralBejarano María Stella, Ruoti Monica, Paredes María Elsa et al . Atención Primaria en Salud en Paraguay: panorámica y perspectiva. Ciênc. saúdecoletiva. 2011 June; 16( 6 ): 2865-2875. Disponible en: http://www.scielo.br/scielo. php?script= sci_arttext\&pid=S1413-

$81 \overline{2} 32011000600024 \&$ Ing $=$ en. https:// doi.org/10.1590/S141381232011000600024.

4. Montañez, C. La demanda por servicios de salud. Una aproximación teórica. Revista de la facultad de ciencias económicas de la UNMSM. 2002; 15170.

5. Sandra Lucía Pérez, S. Arrivillaga, M. Redes integradas de servicios de salud en el marco de la atención primaria en salud en países seleccionados de América Latina Vol. 3 Núm. 2 (2017): Revista Salutem Scientia Spiritus. Disponible en https://revistas.javerianacali.edu.co/in dex.php/salutemscientiaspiritus/article/ view/ 1791

6. Cabral-Bejarano María Stella, Nigenda Gustavo, Arredondo Armando, Conill Eleonor. Rectoría y gobernanza: dimensiones estructurantes para la implementación de Políticas de Atención Primaria de Salud en el Paraguay, 2008-2017. Ciênc. saúde coletiva [Internet]. 2018 July [cited 2020 Aug 11]; 23( 7 ): 22292238. Available from: http://www.scielo.br/scielo.php?script= sci_arttext\&pid=S1413-

$81 \overline{2} 32018000702229 \&$ Ing $=$ en. https:// doi.org/10.1590/1413-

81232018237.09242018.
7. Organización Panamericana de la Salud. La renovación de la atención primaria de salud en las Américas: documento de posición de la Organización Panamericana de la Salud/Organización Mundial de la Salud (OPS/OMS). Washington, DC: OPS; 2007. [ citado 3 enero 2017]. Disponible en:

http: //iris. paho.org/xmlui/bitstream/ha nd le/123456789/31084/9275326991esp.PDF

8. Mallar MA. La Gestión por Procesos: un enfoque de gestión eficiente. Vis. futuro. 2010 J unio; 13(1): 1-23

9. Lerea MJ, Tullo JE, López P. Estrategia de atención primaria de salud y su impacto en las hospitalizaciones evitables por condiciones sensibles a la atención ambulatoria, Paraguay, 20002017 Rev Panam Salud Publica. 2019 Aug 22;43:e 69. Spanish. doi: 10.26633/RPSP.2019.69. PMID: 31456822; PMCID: PMC6705332.

10. Fajardo-Dolci G, Gutiérrez JP, GarcíaSaisó S. Acceso efectivo a los servicios de salud: operacionalizando la cobertura universal en salud. Salud Publica Mex (internet) 2015 (citado 20 marzo 2019); 57: 180-86. Disponible en. http://www.medigraphic.com/pdfs/salp ubmex/sal-2015/sal152j.pdf

11. Organización Panamericana de la Salud. Redes integradas de servicios de salud: conceptos, opciones de política y hoja de ruta para su Implementación en las Américas. (Serie: La Renovación de la Atención Primaria de Salud en las Américas No.4). Washington, DC: OPS; 2010. [citado 3 mayo 2018]. Disponible en:

http: // iris. paho.org/xmlui/bitstream/ha ndle/123456789/31323/978927533116 3-spa.PDF

12. Presidencia de la Republica. Gabinete Social. Transformar nuestro mundo: la Agenda 2030 para el Desarrollo Sostenible. Paraguay, 2015.Disponible en: https://www.gabinetesocial.gov.py/ articulo/321-transformar-nuestromundo-la-agenda-2030-para-eldesarrollo-sostenible.html 
13. Cosavalente-Vidarte Oscar, Zevallos Leslie, Fasanando Jose, Cuba-Fuentes Sofia. Proceso de transformación hacia las redes integradas de salud en el Perú. Rev. perú. med. exp. salud publica [Internet]. 2019 Jun [citado 2020 Jul 22] ; 36( 2 ): 31925.

Disponible

en: http://www.scielo.org.pe/scielo.php ?script $=$ sci_arttext\&pid $=$ S1726-

46342019000200022\&lng=es. http://d x.doi.org/10.17843/rpmesp. 2019.362.4 623.

14. Hirmas Aday M, Poffald Angulo L, Jasmen Sepúlveda AM, Aguilera Sanhueza X, Delgado Becerra I, Vega Morales J. Barreras y facilitadores de acceso a la atención de salud: una revisión sistemática cualitativa [Healthcareaccessbarriers and facilitators: a qualitativesystematicreview]. RevPana m Salud Publica. 2013;33(3):223-29. doi: $10.1590 /$ s1020-

49892013000300009. Disponible en: https://pubmed.ncbi.nlm.nih.gov/2369 8142/

15. Ministerio de Salud Publica y Bienestar Social. Manual de Organización de los Servicios en el marco de las RIISS, Paraguay 2019. Disponible en: https: //www.mspbs.gov.py/dependenci as/planificacion/adjunto/17613eManualdeOrganizacinAprobadoORIGINA L.pdf

16. Vargas- Lorenzo I, Vázquez-Navarrete I, Mogollón-Pérez AS. Acceso a la atención en salud en Colombia. Rev. Salud Pública 2010; 12(5): 701-12.

17. Cabezas, César. Atención médica y de salud en el Perú. Rev. Perú. med. exp. saludpublica, Jun 2019, vol.36, no.2, p. 165-66.

18. Giménez Caballero E, Rodríguez JC, Ocampos G, Flores L. Composición del gasto de bolsillo en el sistema de salud del Paraguay. Mem. Inst. Investig. Cienc. Salud. 2017; 15(3): 64-72.

19. Del Carmen Sara José Carlos. Lineamientos y estrategias para mejorar la calidad de la atención en los servicios de salud. Rev. perú. med. exp. salud pública [Internet]. 2019 Jun [citado 2020 Abr 14] ; 36( 2 ): 28895. Disponible en: http: //www.scielo.org.pe/scielo.php?scr ipt $=$ sci_arttext\&pid=S1726-

46342019000200018\&lng=es. http://d x.doi.org/10.17843/rpmesp.2019.362.4 449.
20. Ugarte-Ubilluz Óscar. Gobernanza y rectoría de la calidad en los servicios de salud en el Perú. Rev. perú. med. exp. salud publica [Internet]. 2019 Jun; 36(2): 296-303. Disponible en:

http:// www.scielo.org. pe/scielo. php?scr ipt $=$ sci arttext\&pid=S1726

46342019000200019\&lng=es. http://d x.doi.org/10.17843/rpmesp.2019.362.4 495.

21. Fernández Lorenzo Angie, Pérez Rico Cristina, Méndez Rojas Vicente Eduardo, Fernández García Carlos, Méndez Rojas Adriana Paola, Calero Morales Santiago. Marketing social y su influencia en la solución de problemas de salud. Rev. Cubana InvestBioméd. $2017.36 \quad$ (3). Disponible en: http://scielo.sld.cu/scielo.php?script=sc i_arttext\&pid=S086403002017000300011\&lng=es.

22. Restrepo-Zea, Jairo Humberto, SilvaMaya, Constanza, Andrade-Rivas, Federico, \& VH-Dover, Robert. Acceso a servicios de salud: análisis de barreras y estrategias en el caso de Medellín, Colombia. Revista Gerencia y Políticas de Salud 2014, 13(27), 24265. Disponible en: https: //dx. doi.org/10.11144/J averiana. rgyps13-27.assa

23. Moscoso Miguel G, Villarreal-Zegarra David, Castillo Ronald, Bellido-Boza Luciana, Mezones-Holguín Edward. Validez y confiabilidad de la escala de satisfacción de los usuarios de consulta médica ambulatoria en Perú. Rev. perú. med. exp. salud pública. 2019 Jun.36(2): 167-177. Disponible en:

http: // www.scielo. org.pe/scielo.php?scr ipt $=$ sci_arttext\&pid=S1726-

46342019000200002\&lng=es. http: //d x.doi.org/10.17843/rpmesp.2019.362.4 621.

24. Dalal K. Evolution and patterns of global health financing 1995-2014: development assistance for health, and government, prepaid private, and outof-pocket health spending in 184 countries. The Lancet. 2017;389 (10083): 1981-2004.

25. Van der Stuyft Patrick, De Vos Pol. La relación entre los niveles de atención constituye un determinante clave de la salud. Rev Cubana Salud Pública. 2008 Dic.; 34(4). Disponible en: http: //scielo.sld.cu/scielo.php?script=sc i_arttext\&pid=S0864-

$\overline{3} 4662008000400014 \&$ lng $=$ es. 